\title{
Absolute deformation potentials of two-dimensional materials
}

\author{
Julia Wiktor* and Alfredo Pasquarello \\ Chaire de Simulation à l'Echelle Atomique, Ecole Polytechnique Fédérale de Lausanne, CH-1015 Lausanne, Switzerland
}

(Received 19 September 2016; revised manuscript received 8 November 2016; published 7 December 2016)

\begin{abstract}
We present $a b$ initio calculations of uniaxial absolute deformation potentials of the valence and the conduction bands in monolayer $\mathrm{MoS}_{2}, \mathrm{MoSe}_{2}, \mathrm{WS}_{2}, \mathrm{WSe} 2, \mathrm{~h}-\mathrm{BN}$, and phosphorene. Calculations are performed using both semilocal and hybrid functionals. The absolute positions of the band edges in strained and unstrained materials are determined using the vacuum level as reference. For $\mathrm{WSe}_{2}$, we compare the obtained results with measured shifts of the valence band maximum (VBM) and the conduction band minimum (CBM) induced by uniaxial strain and find a very good agreement. The parameters describing the shifts in the VBM and CBM positions under strain can be used in the modeling of devices such as tunneling field-effect transistors.
\end{abstract}

DOI: 10.1103/PhysRevB.94.245411

\section{INTRODUCTION}

Two-dimensional (2D) materials, such as graphene [1], phosphorene [2,3], hexagonal boron nitride (h-BN) [4,5], and transition-metal dichalcogenides (TMDs) [6,7], of which $\mathrm{MoS}_{2}, \mathrm{MoSe}_{2}, \mathrm{WS}_{2}$, and $\mathrm{WSe}_{2}$ are among the most studied ones [8-11], have gained significant interest due to their potential applications in future electronics, photovoltaics, and batteries. Recently, strain engineering has become a major focus in the research of 2D materials [12].

Tunneling field-effect transistors (TFETs) [13,14] consisting of vertically stacked graphene and monolayers of hexagonal boron nitride or molybdenum disulphite $\left(\mathrm{MoS}_{2}\right)$ have been realized [15]. In these devices, quantum band-toband tunneling replaces thermal injection, with the tunneling currents depending directly on the band-gap size. This effect was found to be the reason behind the rapid on-off switching capabilities of TFETs, which grants them superiority over conventional FETs. Since the size of the band gap can be tuned by the application of strain, strain engineering is a promising way of adjusting the properties of such devices.

Another interesting example is the recently fabricated 2D phosphorene, which apart from demonstrating excellent performance when applied in a transistor, also possesses a direct band gap, as opposed to graphene, and can be used in optoelectronics. It has recently been shown that a directindirect band-gap transition can be induced by introducing strain in an appropriate direction in this material, making it possible to precisely tune its electronic properties [16].

Hexagonal boron nitride possesses many of the advantageous properties of graphene, while also having a wide direct band gap, making it a much better candidate for being used as a semiconductor in spintronics, where precise control over the magnetic states is necessary. It has been shown that uniaxial strain can be used to finely tune band gaps in zigzag h-BN nanoribbons, while also modifying the magnitude and direction of their dipole moment [17].

Molybdenum disulphide in monolayer form has been successfully applied in FETs, photodetectors, and photovoltaic devices [18], due to its preferential physical properties and

*julia.wiktor@epfl.ch its band gap of $1.8 \mathrm{eV}$. It has recently been demonstrated that by creating a strain profile in an atomically thin layer of $\mathrm{MoS}_{2}$ one could produce a continuously varying band gap, and simultaneously introduce a spatial variation of chargecarrier energies [19]. This effect was proposed to have direct application in photovoltaic devices capable of capturing a broad range of the solar spectrum and to allow the tunneling of energy along the strain gradient.

With the band structure of semiconductors having a direct impact on their applicability and performance in different domains, ways to adjust its properties according to specific needs have been sought intensively. Strain engineering has proven to be an effective way for achieving this goal, even more so in the case of nanostructures, which exhibit a much better tolerance to mechanical strain than their bulk counterparts. Moreover, in devices based on vertical stacking of monolayer materials, strain will be present due to lattice mismatches. Thus, the way that strain affects the material properties needs to be understood. The possibility of controlling device characteristics through strain has led to extensive studies on the way the electronic structure of monolayer materials is affected, both theoretically [16,20-24] and experimentally [2,25-28]. However, most of these studies focused on the band gaps rather than on the positions of the valence- and the conduction-band edges, which are, for instance, necessary to define the band offsets in heterostructures.

In the present study, we investigate uniaxial absolute deformation potentials, which relate the uniaxial strain to the shifts of individual band edges. We consider a set of monolayer materials: $\mathrm{MoS}_{2}, \mathrm{MoSe}_{2}, \mathrm{WS}_{2}, \mathrm{WSe} \mathrm{S}_{2}, \mathrm{~h}-\mathrm{BN}$, and phosphorene. The deformation potentials are important physical properties and are necessary in several applications. For instance, they allow the determination of natural band offsets between lattice mismatched material systems [29], the description of electron-phonon interactions [30], and the definition of pressures in $a b$ initio calculations of charged supercells [31].

This paper is organized as follows. In Sec. II we present the definition of the uniaxial deformation potentials and the computational methods. In Sec. III we describe the calculated absolute deformation potentials and compare them with previous studies. We conclude the paper in Sec. IV. 


\section{METHODS}

\section{A. Uniaxial absolute deformation potentials}

The absolute deformation potentials can be defined as $[32,33]$

$$
a_{i}=\frac{\Delta E_{i}}{\Delta \ln V},
$$

where $a_{i}$ is the deformation potential of a given state $i, \Delta E_{i}$ is the shift of its energy under strain, and $\Delta \ln V=\Delta V / V$ is the relative change of volume. This change can be related to strain through $\Delta V / V=\epsilon_{x x}+\epsilon_{y y}+\epsilon_{z z}$, where $\epsilon_{x x}, \epsilon_{y y}$, and $\epsilon_{z z}$ are the normal strains in $x, y$, and $z$ directions. In the present study, we are interested in uniaxial strains. Therefore, we define uniaxial absolute deformation potentials as

$$
a_{x x}^{i}=\frac{\Delta E_{i}}{\epsilon_{x x}} \quad \text { and } \quad a_{y y}^{i}=\frac{\Delta E_{i}}{\epsilon_{y y}} .
$$

In our electronic structure calculations, we use the vacuum level to align the energies of the electronic states. It is worth noting that in the case of $2 \mathrm{D}$ materials, the vacuum can be easily used as energy reference, as it is present in the simulation cell, while in the case of three-dimensional semiconductors strained superlattice calculations are usually required to calculate absolute deformation potentials [32,33]. From the absolute deformation potentials of the valence and of the conduction band ( $a^{\mathrm{VBM}}$ and $a^{\mathrm{CBM}}$ ), one can directly obtain the band-gap deformation potential $a^{\text {gap }}$ as follows:

$$
a^{\text {gap }}=\frac{\Delta E_{\text {gap }}}{\Delta \ln V}=a^{\mathrm{CBM}}-a^{\mathrm{VBM}} .
$$

\section{B. Computational details}

In the present work, we perform electronic structure calculations using DFT as implemented in the ABINIT code [34-36]. Two types of calculations are performed, one within the semilocal Perdew-Burke-Ernzerhof (PBE) approximation [37] and one using the Perdew-Burke-Ernzerhof (PBE0) hybrid functional [38], in which $25 \%$ of PBE exchange is replaced by nonlocal Fock exchange. Core-valence interactions in all calculations are treated through norm-conserving pseudopotentials [39]. In the calculations for TMDs, we include spin-orbit coupling as calculated at the PBE level. For each material, we use a plane-wave cutoff and a $k$-point sampling that ensure the convergence of the total energy to less than $1 \mathrm{meV}$. This corresponds to cutoff energies of $24 \mathrm{Ha}$ for phosphorene; $38 \mathrm{Ha}$ for $\mathrm{MoS}_{2}, \mathrm{WS}_{2}$, and h-BN; and $40 \mathrm{Ha}$ for $\mathrm{MoSe}_{2}$ and $\mathrm{WSe}_{2}$. We use $12 \times 12 \times 1 k$-point meshes for the TMDs and for h-BN and a $14 \times 10 \times 1 k$-point mesh for phosphorene. Before studying the effects of strain on the band edges, we relax all structures at the PBE level. The equilibrium lattice parameters are found to be $a=3.19 \AA$ for $\mathrm{MoS}_{2}$, $a=3.32 \AA$ for $\mathrm{MoSe}_{2}, a=3.19 \AA$ for $\mathrm{WS}_{2}, a=3.32 \AA$ for $\mathrm{WSe}_{2}, a=2.51 \AA$ for h-BN, and $a=3.30 \AA$ and $b=4.63 \AA$ for phosphorene. We then strain the geometries by setting the lattice parameter in a given direction to a fixed value $(1 \%$ smaller and larger), while allowing the atomic positions and the other lattice parameter to relax. Relaxation is continued until the atomic forces become lower than $0.003 \mathrm{eV} / \AA$. The PBE0 calculations are performed using the PBE structures.
We check in the case of $\mathrm{MoS}_{2}$ that accounting for the relaxations at the PBE0 level leads to changes in the absolute deformation potentials of less than $0.1 \mathrm{eV}$. We introduce a vacuum layer of at least $20 \AA$ between the layers in the simulation cell. We check that this thickness of the vacuum layer allows us to obtain the band-edge positions converged to less than $0.01 \mathrm{eV}$ and absolute deformation potentials to less than $0.2 \mathrm{eV}$.

To verify the consistency of our calculations with previous studies, we analyze the band gaps calculated for the unstrained materials. Using the PBE functional, we obtain 1.60, 1.34, 1.54, 1.26, 4.70, and $0.90 \mathrm{eV}$ for $\mathrm{MoS}_{2}, \mathrm{MoSe}_{2}, \mathrm{WS}_{2}$, $\mathrm{WSe}_{2}, \mathrm{~h}-\mathrm{BN}$, and phosphorene, respectively. These values are consistent with previous electronic structure calculations for 2D materials using semilocal functionals [16,40,41]. Our PBE0 calculations predict band gaps of 2.71, 2.42, 2.64, 2.32, 6.35, and $2.22 \mathrm{eV}$ for $\mathrm{MoS}_{2}, \mathrm{MoSe}_{2}, \mathrm{WS}_{2}, \mathrm{WSe} \mathrm{H}_{2}, \mathrm{~h}-\mathrm{BN}$, and phosphorene, respectively. These values are in good agreement with values obtained using the many-body perturbation theory in the $G W$ approximation [41-43]. As in this work we focus on the positions of the band edges and not only on the band gaps, it is worth noting that when the PBE0 functional predicts correct band gaps, it also yields ionization potentials in excellent agreement with experiments [44]. This supports the use of this method to study the effect of strain on the band energies.

The absolute deformation potentials are the first derivatives of the band energy with respect to strain at equilibrium. Therefore, they can only be used to predict the band energies in a range of strains for which the relationship between them is linear. We determine the linear regime of this dependence in the case of $\mathrm{MoS}_{2}$. The energies of the VBM at the $\mathrm{K}$ and $\Gamma$ points and of the $\mathrm{CBM}$ at the $\mathrm{K}$ and $\Lambda$ points (see Fig. 1 for the positions of these points in the Brillouin zone) as a function of strain are presented in Fig. 2. The results indicate that the relation can be assumed linear for strains between $-5 \%$ (compressive strain) and $+5 \%$ (tensile strain).

\section{RESULTS AND DISCUSSION}

\section{A. Transition-metal dichalcogenides}

First, we study the absolute deformation potentials of TMDs. In Table I, we present the absolute deformation potentials for $\mathrm{MoS}_{2}, \mathrm{MoSe}_{2}, \mathrm{WS}_{2}$, and $\mathrm{WSe}_{2}$, calculated for the local extrema in the valence band and in the conduction band, and for the corresponding band gaps. For all the considered TMDs, the strain induced shifts in the band positions are found to be very similar for $x$ and $y$ directions. Therefore, in these cases, we present PBE0 results only for $\epsilon_{x x}$. For the TMDs, we plot in Fig. 3 the energies (with respect to the vacuum level) of the VBM at the $\mathrm{K}$ and $\Gamma$ points and of the CBM at the $\mathrm{K}$ and $\Lambda$ points as a function of the uniaxial strain in the $x$ direction. The latter are calculated using the absolute deformation potentials as obtained with the PBE and PBE0 functionals. The four TMDs, which share the same hexagonal structure, allow us to compare the effect of strain on the their band-edge positions. It can be observed that the band edges of $\mathrm{MoS}_{2}, \mathrm{MoSe}_{2}, \mathrm{WS}_{2}$, and $\mathrm{WSe} \mathrm{C}_{2}$ shift under strain in a similar way. For all these materials the VBM and CBM energies at the $\mathrm{K}$ point decrease when the lattice parameter increases, while the VBM energy at $\Gamma$ and the CBM energy at $\Lambda$ increase. 
$\mathrm{MoS}_{2}$

(a)

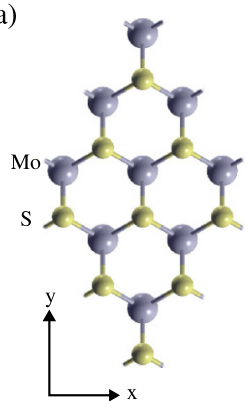

(b)

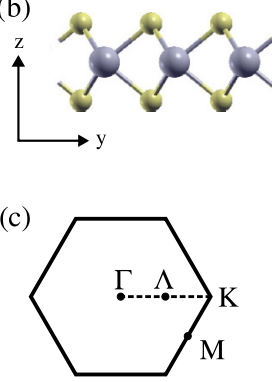

(d)

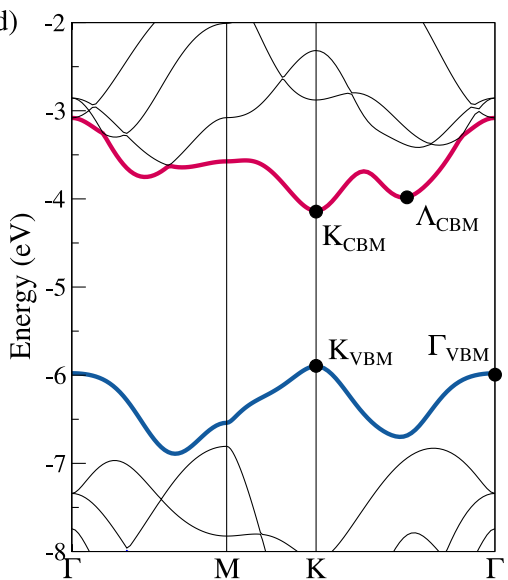

$h$-BN

(b)
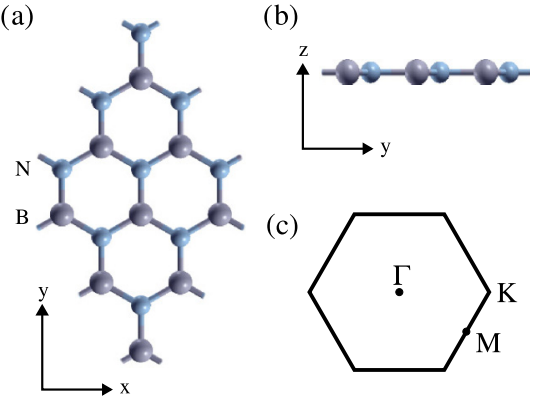

(c)

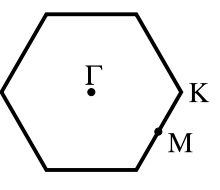

(d)

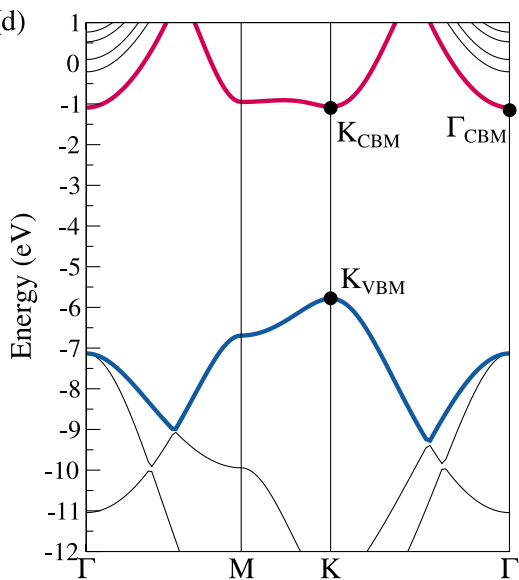

Phosphorene

(b)

(a)
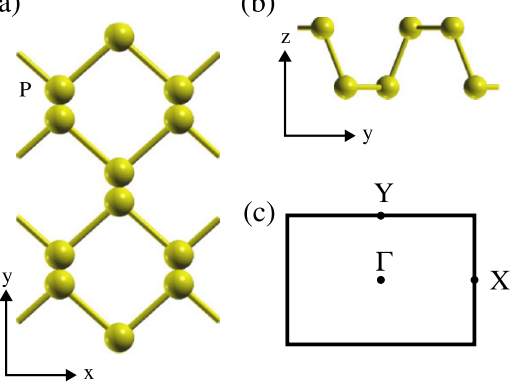

(d)

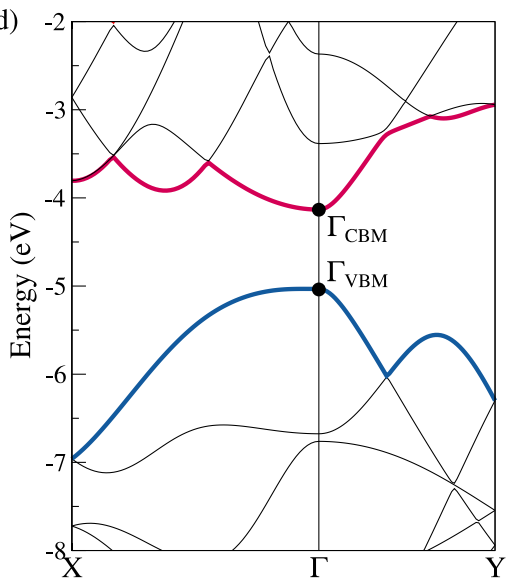

FIG. 1. Top (a) and side (b) views of the structures of $\mathrm{MoS}_{2}$, h-BN, and phosphorene along with the representations of the Brillouin zones (c) and of the band structures calculated with the PBE functional (d). In (d), the points for which the absolute deformation potentials are calculated are indicated. Band structures are aligned to the vacuum level.

The absolute changes of the VBM and CBM energies under strain are due to two effects. First, the kinetic energy of the state, which is proportional to the reciprocal-lattice vector, decreases when the lattice parameter of the material is increased [45]. Second, the state can be either bonding

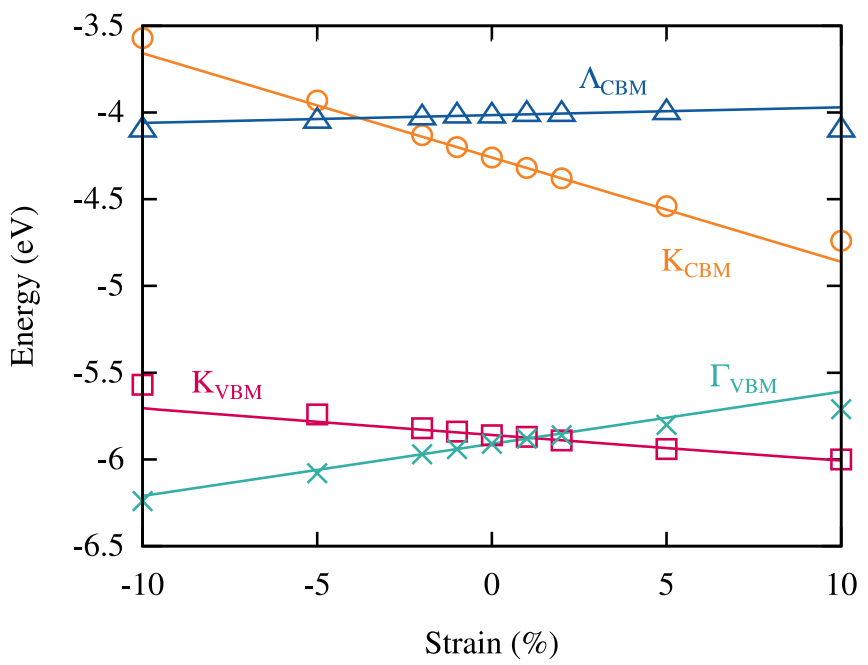

FIG. 2. Energies of the VBM at the $\mathrm{K}$ and $\Gamma$ points and of the $\mathrm{CBM}$ at the $\mathrm{K}$ and $\Lambda$ points of $\mathrm{MoS}_{2}$ as a function of the uniaxial strain in the $x$ direction. Squares represent calculated values and solid lines are linear fits using values calculated for strains of +1 and $-1 \%$. The vacuum energy is used as reference. or antibonding, which will result in a respective increase or decrease of its energy level as the bond length is increased. For antibonding states the two effects add up, resulting in large negative absolute deformation potentials. In the case of bonding states, the two effects lead to partial compensation, resulting in lower values of the corresponding deformation potentials. In TMDs, the CBM state at the $\mathrm{K}$ point is a metal $d_{z^{2}}$-like function showing antibonding character with the chalcogen $p_{x}+p_{y}$ orbitals. The energy of this state strongly decreases when the material is expanded. The VBM state at the $\Gamma$ point consists of strongly bonded metal $d_{z^{2}}$ and chalcogen $p_{z}$ orbitals. In this case we observe a positive deformation potential, but with a lower value than for the $\mathrm{CBM}$ at the $\mathrm{K}$ point state, since the bonding effect is partially compensated by the kinetic-energy effect. The VBM at the $\mathrm{K}$ point is a bonding state between metal $d_{x y}$ and chalcogen $p_{x}+p_{y}$ orbitals. The CBM at the $\Delta$ point is a metal $d_{x^{2}-y^{2}}$-like wave function showing bonding character with the chalcogen $p_{x}+p_{y}$ orbitals. For these two states we observe small deformation potentials due to the two competing effects.

Our PBE results are in good agreement with results obtained in the local density approximation by Huang et al. [27]. Even though the absolute deformation potentials are not extracted or discussed, we can estimate them from Fig. 5 of their work [27]. For instance, for the VBM and the CBM at the $\mathrm{K}$ point they obtain uniaxial absolute deformation potentials in the $x$ direction of about -2.5 and $-7.5 \mathrm{eV}$, respectively. These values are in qualitative agreement with the values of 
TABLE I. Absolute deformation potentials (in $\mathrm{eV}$ ) of the $\mathrm{VBM}$ at the $\mathrm{K}$ and $\Gamma$ points, of the $\mathrm{CBM}$ at the $\mathrm{K}$ and $\Lambda$ points and of the various band gaps of monolayer transition-metal dichalcogenides.

\begin{tabular}{|c|c|c|c|c|c|c|c|c|}
\hline & & $a_{\mathrm{K}}^{\mathrm{VBM}}$ & $a_{\mathrm{K}}^{\mathrm{CBM}}$ & $\begin{array}{c}\mathrm{MoS}_{2} \\
a_{\Gamma}^{\mathrm{VBM}}\end{array}$ & $a_{\Lambda}^{\mathrm{CBM}}$ & $a_{\mathrm{K}-\mathrm{K}}^{\mathrm{gap}}$ & $a_{\Gamma-\mathrm{K}}^{\text {gap }}$ & $a_{\mathrm{K}-\Lambda}^{\text {gap }}$ \\
\hline PBE & $x x$ & -1.86 & -6.08 & 3.13 & 0.61 & -4.22 & -9.21 & 2.48 \\
\hline PBE & yy & -1.93 & -6.19 & 2.90 & 0.15 & -4.26 & -9.09 & 2.08 \\
\hline \multirow[t]{2}{*}{ PBE0 } & $x x$ & -1.71 & -7.16 & 3.12 & 0.63 & -5.42 & -10.29 & 2.34 \\
\hline & & $a_{\mathrm{K}}^{\mathrm{VBM}}$ & $a_{\mathrm{K}}^{\mathrm{CBM}}$ & $\begin{array}{l}\mathrm{MoSe}_{2} \\
a_{\Gamma}^{\mathrm{VBM}}\end{array}$ & $a_{\Lambda}^{\mathrm{CBM}}$ & $a_{\mathrm{K}-\mathrm{K}}^{\mathrm{gap}}$ & $a_{\Gamma-\mathrm{K}}^{\mathrm{gap}}$ & $a_{\mathrm{K}-\Lambda}^{\text {gap }}$ \\
\hline PBE & $x x$ & -1.86 & -5.62 & 2.55 & 0.71 & -3.75 & -8.16 & 2.57 \\
\hline \multirow[t]{2}{*}{ PBE0 } & $x x$ & -1.27 & -6.21 & 3.13 & 0.99 & -4.95 & -9.33 & 2.26 \\
\hline & & $a_{\mathrm{K}}^{\mathrm{VBM}}$ & $a_{\mathrm{K}}^{\mathrm{CBM}}$ & $\begin{array}{c}\mathrm{WS}_{2} \\
a_{\Gamma}^{\mathrm{VBM}} \\
\end{array}$ & $a_{\Lambda}^{\mathrm{CBM}}$ & $a_{\mathrm{K}-\mathrm{K}}^{\mathrm{gap}}$ & $a_{\Gamma-\mathrm{K}}^{\text {gap }}$ & $a_{\mathrm{K}-\Lambda}^{\mathrm{gap}}$ \\
\hline$\overline{\mathrm{PBE}}$ & $x x$ & -1.59 & -6.76 & 3.09 & 0.90 & -5.17 & -9.85 & 2.49 \\
\hline \multirow[t]{2}{*}{ PBE0 } & $x x$ & -1.43 & -7.73 & 3.68 & 1.01 & -6.30 & -11.40 & 2.44 \\
\hline & & $a_{\mathrm{K}}^{\mathrm{VBM}}$ & $a_{\mathrm{K}}^{\mathrm{CBM}}$ & $\begin{array}{c}\mathrm{WSe}_{2} \\
a_{\Gamma}^{\mathrm{VBM}}\end{array}$ & $a_{\Lambda}^{\mathrm{CBM}}$ & $a_{\mathrm{K}-\mathrm{K}}^{\text {gap }}$ & $a_{\Gamma-\mathrm{K}}^{\mathrm{gap}}$ & $a_{\mathrm{K}-\Lambda}^{\text {gap }}$ \\
\hline$\overline{\mathrm{PBE}}$ & $x x$ & -1.43 & -6.35 & 2.61 & 0.93 & -4.92 & -8.97 & 2.36 \\
\hline PBE0 & $x x$ & -0.16 & -6.03 & 4.06 & 1.13 & -5.86 & -10.08 & 1.30 \\
\hline
\end{tabular}

-1.86 and $-6.08 \mathrm{eV}$ calculated within the PBE functional in the present study. Differences amount to about 20-30\%.

Next, we compare the results achieved with the PBE and PBE0 functionals. The two methods predict similar absolute deformation potentials in most of cases. The largest difference is observed for the CBM at the $\mathrm{K}$ point. In the case of $\mathrm{WSe}_{2}$, we also observe a significant difference for the VBM at the $\mathrm{K}$ point. However, as for the positions of the band edges (see
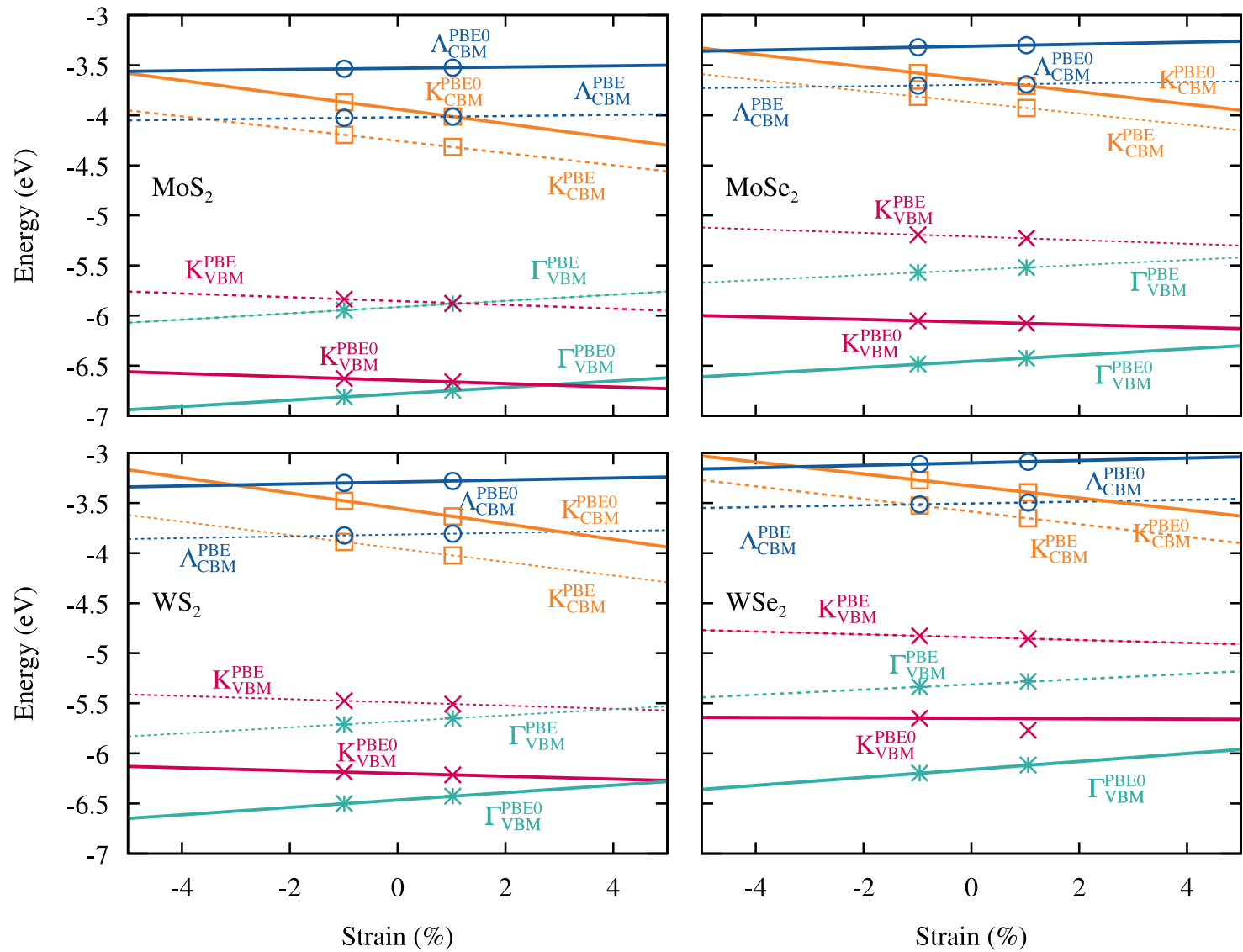

FIG. 3. Energies of the VBM at the $\mathrm{K}$ and $\Gamma$ points and of the $\mathrm{CBM}$ at the $\mathrm{K}$ and $\Lambda$ points of the transition-metal dichalcogenides as a function of the uniaxial strain in the $x$ direction. Solid and dashed lines correspond to PBE0 and PBE results, respectively. The vacuum energy is used as reference. The band energies result from a linear extrapolation of the values calculated at -1 and $+1 \%$ strain, indicated with symbols. 
TABLE II. Absolute deformation potentials (in eV) of the VBM at the $\mathrm{K}$ point, of the $\mathrm{CBM}$ at the $\mathrm{K}$ and $\Gamma$ points, and of the band gaps of monolayer hexagonal boron nitride.

\begin{tabular}{lllllll}
\hline \hline & & \multicolumn{5}{c}{$\mathrm{h}-\mathrm{BN}$} \\
& & $a_{\mathrm{K}}^{\mathrm{VBM}}$ & $a_{\mathrm{K}}^{\mathrm{CBM}}$ & $a_{\Gamma}^{\mathrm{CBM}}$ & $a_{\mathrm{K}-\mathrm{K}}^{\mathrm{gap}}$ & $a_{\mathrm{K}-\Gamma}^{\text {gap }}$ \\
\hline PBE & $x x$ & -1.63 & -4.67 & -0.51 & -3.05 & 1.12 \\
PBE & $y y$ & -1.56 & -4.62 & -0.49 & -3.06 & 1.07 \\
PBE0 & $x x$ & -1.52 & -4.71 & -0.35 & -3.18 & 1.17 \\
\hline
\end{tabular}

Fig. 3) PBE0 yields much larger regions in which the band gap is direct. For instance, in the case of $\mathrm{MoS}_{2}$ the PBE functional predicts a direct band gap for strains between -3.5 and $+1.2 \%$, while the PBE0 functional yields such a result in the range from -5 to $+2.8 \%$.

\section{B. Hexagonal boron nitride}

We consider here monolayer hexagonal boron nitride. Table II presents the absolute deformation potentials of the VBM at the $\mathrm{K}$ point and of the $\mathrm{CBM}$ at the $\mathrm{K}$ and $\Gamma$ points of this material. No significant differences are found between the effects of strain in the $x$ and $y$ directions, nor between the PBE and PBE0 results. However, similar to the case of TMDs, the direct/indirect nature of the band gap differs between PBE and PBE0 calculations, as can be seen in Fig. 4. While PBE predicts a transition between an indirect and direct gap at a strain of about $-0.5 \%$, an indirect gap is observed in PBE0 for all strains between -5 and $+5 \%$. Using both the PBE and PBE0 functionals, we find that the energies of the three considered states decrease when the lattice parameter increases. The evolution of the band edges as a function of strain can be related to the chemical bonding of the corresponding states.

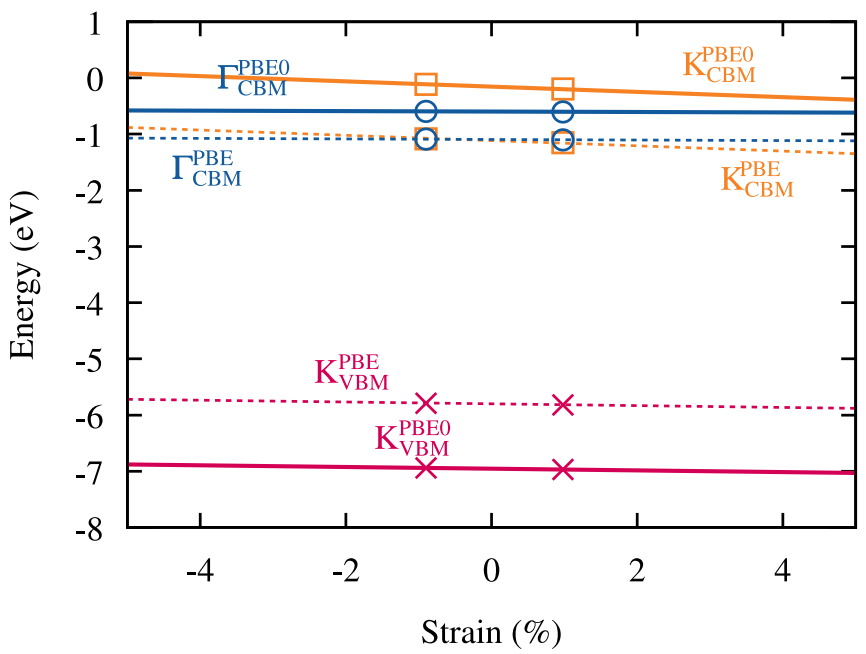

FIG. 4. Energies of the VBM at the $\mathrm{K}$ and $\Gamma$ points and of the $\mathrm{CBM}$ at the $\mathrm{K}$ point of monolayer hexagonal boron nitride as a function of the uniaxial strain in the $x$ direction. Solid and dashed lines correspond to PBE0 and PBE results, respectively. The vacuum energy is used as reference. The band energies result from a linear extrapolation of the values calculated at -1 and $+1 \%$ strain, indicated with symbols.
TABLE III. Absolute deformation potentials (in eV) of the VBM, of the $\mathrm{CBM}$, and of the band gap at the $\Gamma$ point for phosphorene.

\begin{tabular}{llccc}
\hline \hline & $\begin{array}{c}\text { Phosphorene } \\
a_{\Gamma}^{\text {VBM }}\end{array}$ & $a_{\Gamma}^{\mathrm{CBM}}$ & $a_{\Gamma-\Gamma}^{\text {gap }}$ \\
\hline PBE & $x x$ & 1.94 & 4.17 & 2.23 \\
PBE & $y y$ & -2.93 & 0.53 & 3.46 \\
PBE0 & $x x$ & 1.47 & 4.66 & 3.20 \\
PBE0 & $y y$ & -3.04 & 0.54 & 3.59 \\
\hline \hline
\end{tabular}

At the $\mathrm{K}$ point, the VBM and the CBM correspond to $\pi$ (bonding) and $\pi^{*}$ (antibonding) states, respectively [46]. For the bonding state at the VBM, we observe a small negative absolute deformation potential, because the increase of its energy level with bond length is dominated by the decrease of the kinetic energy. For the antibonding CBM state, the energy decreases when the material is expanded. This adds up to the kinetic energy decrease, resulting in a larger negative deformation potential. At the $\Gamma$ point, the energy level of the CBM remains almost unchanged when strain is applied. This is because this state displays a nearly-free-electron-like character [47] and is thus almost unaffected by interatomic distances. It is also interesting to notice that the indirect $\mathrm{K}-\Gamma$ band gap of h-BN does not change significantly when strain is applied, as both band edges move in the same direction.

\section{Phosphorene}

Finally, we study the strain effects in phosphorene. The absolute deformation potentials of the VBM and of the CBM at the $\Gamma$ point of this material are presented in Table III. It is worth noting that under strain, the VBM of phosphorene continuously evolves from the $\Gamma$ point towards the $X$ point [16] (see Fig. 1 for the representation of the Brillouin zone) resulting in an indirect gap. However, in the range of strains that we consider, the maximum of the valence band does not differ from the energy at the $\Gamma$ point by more than $0.02 \mathrm{eV}$. Therefore, we only present results for the $\Gamma$ point in Table III and Fig. 5.

In phosphorene, the effect of strain is significantly different between the $x$ and the $y$ direction, which is consistent with the anisotropic nature of this material [2]. In this case, we present the PBE0 results for both $\epsilon_{x x}$ and $\epsilon_{y y}$. The energies of the VBM and CBM at the $\Gamma$ point as a function of strain are shown in Fig. 5. When a tensile strain is applied in the $x$ direction, both the VBM and the CBM shift upwards in energy. When the material is expanded in the $y$ direction, the CBM remains almost constant, while the VBM decreases. However, while the changes in the individual band edges are different for the strain applied along the $x$ and $y$ directions, the overall effect on the band gap does not depend on the direction in which the strain is applied. For phosphorene, the evolution of the VBM and CBM levels with strain has already been studied by Peng et al. [16]. In this study, the absolute deformation potentials were not calculated. However, we can estimate them from the shift in the VBM and the CBM presented there. For uniaxial strain in the $x$ direction, Peng et al. obtained $a_{\Gamma}^{\mathrm{VBM}}$ and $a_{\Gamma}^{\mathrm{CBM}}$ of about 0.9 and $3.1 \mathrm{eV}$, respectively. Respective values for the $y$ direction amount to -1.0 and $2.8 \mathrm{eV}$. The 

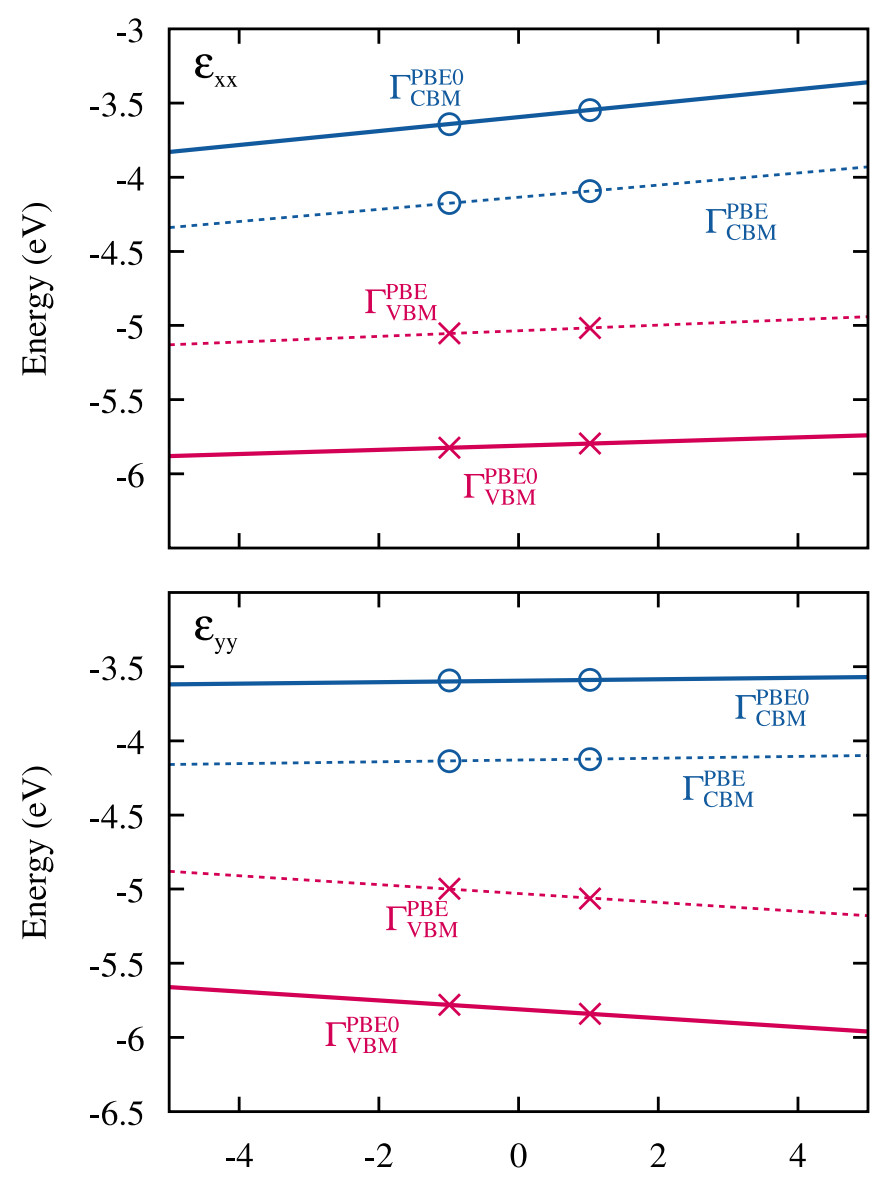

FIG. 5. Energies of the VBM and of the CBM at the $\Gamma$ point of the phosphorene as a function of uniaxial strain in the $x$ and in the $y$ direction. Solid and dashed lines correspond to PBE0 and PBE results, respectively. The vacuum energy is used as reference. The band energies result from a linear extrapolation of the values calculated at -1 and $+1 \%$ strain, indicated with symbols.

corresponding $x x$ and $y y$ band-gap deformation potentials are 2.2 and $3.8 \mathrm{eV}$. While the absolute deformation potentials of the VBM and the CBM estimated from the study of Peng et al. differ from the values reported here by about $1 \mathrm{eV}$, the general trends in the shifts of the band edges and in the band-gap deformation potentials are in good agreement. In Ref. [16], the authors also analyzed the nature of the band-edge states of phosphorene at the $\Gamma$ point. They suggested that the VBM state has a nonbonding and antibonding character in the $x$ and $y$ direction, respectively, while the respective characters of the CBM state are bonding and antibonding. This analysis explains well the uniaxial absolute deformation potentials presented here.

The shifts in the VBM and the CBM of phosphorene were also studied by $\mathrm{Sa}$ et al. [48]. From their results, we extract a deformation potential $a_{\Gamma}^{\mathrm{VBM}}$ of about -0.4 and $-3.7 \mathrm{eV}$ in the $x$ and $y$ directions, respectively. Respective values for $a_{\Gamma}^{\mathrm{CBM}}$ are 5.9 and $1.3 \mathrm{eV}$. Most of the deformation potentials are in qualitative agreement with the ones presented here. However, we notice that in the case of $a_{\Gamma}^{\mathrm{VBM}}$ in the $x$ direction Sa et al. found a result with an opposite sign, with a value that differs by more than $2 \mathrm{eV}$ from the one reported here.

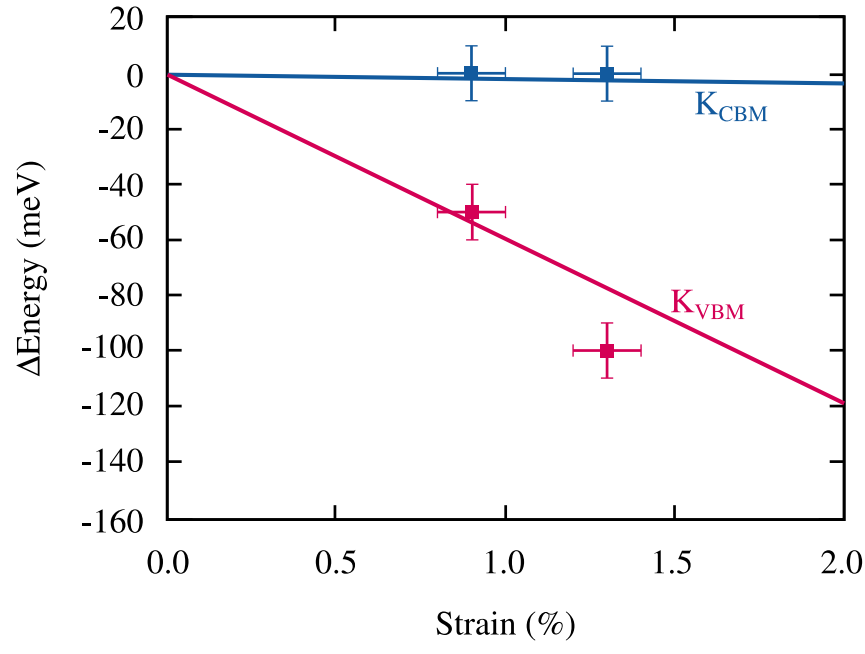

FIG. 6. Comparison between the energy shifts of the VBM and $\mathrm{CBM}$ of $\mathrm{WSe}_{2}$, as a function of uniaxial strain, as extracted from experiments [28] (red and blue squares, respectively) and calculated with the PBE0 functional in the present study.

\section{Comparison with experiments}

The absolute deformation potentials presented in this work can be compared with experimental data. Several photoluminescence spectroscopy measurements of the band-gap evolution under uniaxial strain have been performed for $\mathrm{MoS}_{2}[25,49,50]$. In these studies, a decrease of the direct gap energy of 45-70 meV/\% strain was observed. Our band-gap deformation potentials of -4.22 and $-5.42 \mathrm{eV}$ (corresponding to -42.2 and $-54.2 \mathrm{meV} / \%$ strain) calculated using the PBE and $\mathrm{PBE} 0$ functional, respectively, are within the ranges of the experimental values.

Recently, the strain effect on the band edges of $\mathrm{WSe}_{2}$ has been studied experimentally through the realization of a three terminal FET [28]. It was observed that under uniaxial tensile strain the valence-band edge remains almost unchanged, while the conduction-band edge moves down in energy. In Fig. 6, we compare the shifts in the VBM and CBM, as calculated with the PBE0 functional, with experimental data from Ref. [28]. A good agreement is found between the calculated and measured energy shifts. It is worth noting that in Ref. [28] the results were compared to calculations performed for $\mathrm{MoS}_{2}$ under strain [20], in which all band structures were aligned to the VBM. This means that in Ref. [20] the energy of the valence band was artificially set to zero for all strains. The agreement invoked in Ref. [28] could thus only be achieved due to the negligible shift of the VBM, as this becomes clear through the calculations in this work.

For future applications of the absolute deformation potentials presented here, it should be kept in mind that monolayer materials are often placed on a substrate or embedded in 2D heterostructures. It is, therefore, worth discussing the applicability of the absolute deformation potentials calculated here in these conditions. It has been shown experimentally [51] that when monolayer $\mathrm{MoS}_{2}$ is deposited directly on $\mathrm{Si}$ and $\mathrm{SiO}_{2}$ substrates, it exhibits a warped surface morphology, which deteriorates its optoelectronic properties. In this case, the absolute deformation potentials presented in this study 
cannot be applied directly. However, in the same study, it was shown that when $\mathrm{MoS}_{2}$ is placed on an atomically thin buffer layer of h-BN, its electronic properties are recovered, since the interaction with the substrate is weak. Similarly, first-principles calculations of monolayer $\mathrm{MoS}_{2}$ on h-BN [52] showed that the band structure of the heterostructure is equivalent to the superposition of the band structures of the isolated materials, when an appropriate strain is considered. This suggests that when the interlayer interactions are weak, the absolute deformation potentials calculated in this work could be reliably used to predict band-edge positions in strained heterostructures or in strained supported monolayers.

\section{CONCLUSIONS}

We performed ab-initio density functional theory calculations of the absolute deformation potentials for a set of monolayer materials: $\mathrm{MoS}_{2}, \mathrm{MoSe}_{2}, \mathrm{WS}_{2}, \mathrm{WSe}$, h-BN, and phosphorene. The absolute deformation potentials allow one to directly obtain the energy shifts of the individual band edges upon application of strain. Absolute deformation potentials are required to determine natural band offsets in lattice mismatched material systems, to describe electron-phonon interactions, and to define pressures in ab-initio calculations of charged supercells. The calculations were performed using both the semilocal PBE functional and the hybrid PBE0 functional. The vacuum energy was used to align the energies of the electronic states. For the transition-metal dichalcogenides, we found that the hybrid functional yields much larger ranges of strain with a direct band gap than the PBE functional does. Also, the PBE0 functional predicts hexagonal boron nitride to have an indirect gap for all strains between -5 and $+5 \%$, while the PBE functional yields a transition between an indirect and direct gap around $-0.5 \%$ strain. We observed that for $\mathrm{h}-\mathrm{BN}$ the energies of both VBM and CBM decrease when the lattice parameter increases, resulting in a very small variation of the band gap under strain. In the case of phosphorene, the PBE and PBE0 functionals give similar qualitative and quantitative results. We compared our PBE0 results with a recent experimental study of the strain effect on the band edges of $\mathrm{WSe}_{2}$ [28], finding a very good agreement.

Our study demonstrates the capability of DFT calculations to precisely determine the subtle changes induced by strain in the band-edge positions of 2D materials. The obtained results can be readily applied to device design and can facilitate the development of strain engineering, which has become a major focus in the materials science of 2D systems.

\section{ACKNOWLEDGMENTS}

Financial support is acknowledged from the Swiss National Science Foundation (Grant No. 200020-152799). We used computational resources of Swiss National Supercomputing Centre and École polytechnique fdrale de Lausanne.
[1] K. Novoselov, A. K. Geim, S. Morozov, D. Jiang, M. Katsnelson, I. Grigorieva, S. Dubonos, and A. Firsov, Nature (London) 438, 197 (2005).

[2] H. Liu, A. T. Neal, Z. Zhu, Z. Luo, X. Xu, D. Tománek, and P. D. Ye, ACS Nano 8, 4033 (2014).

[3] F. Xia, H. Wang, and Y. Jia, Nat. Commun. 5, 4458 (2014).

[4] K. Watanabe, T. Taniguchi, and H. Kanda, Nat. Mater. 3, 404 (2004).

[5] D. Pacile, J. Meyer, C. O. Girit, and A. Zettl, App. Phys. Lett. 92, 133107 (2008).

[6] P. Joensen, R. Frindt, and S. R. Morrison, Mater. Res. Bull. 21, 457 (1986).

[7] J. Wilson and A. Yoffe, Adv. Phys. 18, 193 (1969).

[8] Q. H. Wang, K. Kalantar-Zadeh, A. Kis, J. N. Coleman, and M. S. Strano, Nat. Nanotechnology 7, 699 (2012).

[9] W. Zhao, Z. Ghorannevis, L. Chu, M. Toh, C. Kloc, P.-H. Tan, and G. Eda, ACS nano 7, 791 (2012).

[10] Y.-H. Lee, L. Yu, H. Wang, W. Fang, X. Ling, Y. Shi, C.-T. Lin, J.-K. Huang, M.-T. Chang, C.-S. Chang et al., Nano Lett. 13, 1852 (2013).

[11] K. F. Mak and J. Shan, Nat. Photonics 10, 216 (2016).

[12] R. Roldán, A. Castellanos-Gomez, E. Cappelluti, and F. Guinea, J. Phys.: Condens. Matter 27, 313201 (2015).

[13] J. Appenzeller, Y.-M. Lin, J. Knoch, and P. Avouris, Phys. Rev. Lett. 93, 196805 (2004).

[14] B. Radisavljevic, A. Radenovic, J. Brivio, V. Giacometti, and A. Kis, Nat. Nano. 6, 147 (2011).

[15] L. Britnell, R. V. Gorbachev, R. Jalil, B. D. Belle, F. Schedin, A. Mishchenko, T. Georgiou, M. I. Katsnelson, L. Eaves, S. V. Morozov et al., Science 335, 947 (2012).
[16] X. Peng, Q. Wei, and A. Copple, Phys. Rev. B 90, 085402 (2014).

[17] J. Qi, X. Qian, L. Qi, J. Feng, D. Shi, and J. Li, Nano Lett. 12, 1224 (2012).

[18] X. Li and H. Zhu, J. Materiomics 1, 33 (2015).

[19] J. Feng, X. Qian, C.-W. Huang, and J. Li, Nat. Photon. 6, 866 (2012).

[20] P. Johari and V. B. Shenoy, ACS Nano 6, 5449 (2012).

[21] W. S. Yun, S. W. Han, S. C. Hong, I. G. Kim, and J. D. Lee, Phys. Rev. B 85, 033305 (2012).

[22] H. Shi, H. Pan, Y.-W. Zhang, and B. I. Yakobson, Phys. Rev. B 87, 155304 (2013).

[23] A. S. Rodin, A. Carvalho, and A. H. Castro Neto, Phys. Rev. Lett. 112, 176801 (2014).

[24] H. Y. Lv, W. J. Lu, D. F. Shao, and Y. P. Sun, Phys. Rev. B 90, 085433 (2014).

[25] H. J. Conley, B. Wang, J. I. Ziegler, R. F. Haglund, Jr., S. T. Pantelides, and K. I. Bolotin, Nano Lett. 13, 3626 (2013).

[26] A. Castellanos-Gomez, R. Roldán, E. Cappelluti, M. Buscema, F. Guinea, H. S. van der Zant, and G. A. Steele, Nano Lett. 13, 5361 (2013).

[27] Y. L. Huang, Y. Chen, W. Zhang, S. Y. Quek, C.-H. Chen, L.-J. Li, W.-T. Hsu, W.-H. Chang, Y. J. Zheng, W. Chen and A. T. S. Wee, Nat. Commun. 6, 6298 (2015).

[28] T. Shen, A. V. Penumatcha, and J. Appenzeller, ACS Nano 10, 4712 (2016).

[29] Y.-H. Li, A. Walsh, S. Chen, W.-J. Yin, J.-H. Yang, J. Li, J. L. Da Silva, X. Gong, and S.-H. Wei, App. Phys. Lett. 94, 212109 (2009).

[30] J. Bardeen and W. Shockley, Phys. Rev. 80, 72 (1950). 
[31] F. Bruneval, C. Varvenne, J.-P. Crocombette, and E. Clouet, Phys. Rev. B 91, 024107 (2015).

[32] C. G. Van de Walle and R. M. Martin, Phys. Rev. Lett. 62, 2028 (1989).

[33] R. Resta, L. Colombo, and S. Baroni, Phys. Rev. B 41, 12358 (1990).

[34] X. Gonze, J.-M. Beuken, R. Caracas, F. Detraux, M. Fuchs, G.-M. Rignanese, L. Sindic, M. Verstraete, G. Zerah, F. Jollet et al., Comput. Mater. Sci. 25, 478 (2002).

[35] X. Gonze, B. Amadon, P.-M. Anglade, J.-M. Beuken, F. Bottin, P. Boulanger, F. Bruneval, D. Caliste, R. Caracas, M. Côté et al., Comput. Phys. Commun. 180, 2582 (2009).

[36] X. Gonze, F. Jollet, F. A. Araujo, D. Adams, B. Amadon, T. Applencourt, C. Audouze, J.-M. Beuken, J. Bieder, A. Bokhanchuk et al., Comput. Phys. Commun. 205, 106 (2016).

[37] J. P. Perdew, K. Burke, and M. Ernzerhof, Phys. Rev. Lett. 77, 3865 (1996).

[38] J. P. Perdew, M. Ernzerhof, and K. Burke, J. Chem. Phys. 105, 9982 (1996).

[39] D. R. Hamann, Phys. Rev. B 88, 085117 (2013).

[40] J. Kang, S. Tongay, J. Zhou, J. Li, and J. Wu, App. Phys. Lett. 102, 012111 (2013).
[41] H. Şahin, S. Cahangirov, M. Topsakal, E. Bekaroglu, E. Akturk, R. T. Senger, and S. Ciraci, Phys. Rev. B 80, 155453 (2009).

[42] H.-P. Komsa and A. V. Krasheninnikov, Phys. Rev. B 86, 241201 (2012).

[43] V. Tran, R. Soklaski, Y. Liang, and L. Yang, Phys. Rev. B 89, 235319 (2014).

[44] W. Chen and A. Pasquarello, Phys. Rev. B 90, 165133 (2014).

[45] S.-H. Wei and A. Zunger, Phys. Rev. B 60, 5404 (1999).

[46] M. Topsakal, E. Aktürk, and S. Ciraci, Phys. Rev. B 79, 115442 (2009).

[47] X. Blase, A. Rubio, S. G. Louie, and M. L. Cohen, Phys. Rev. B 51, 6868 (1995).

[48] B. Sa, Y.-L. Li, J. Qi, R. Ahuja, and Z. Sun, J. Phys. Chem. C 118, 26560 (2014).

[49] C. Zhu, G. Wang, B. Liu, X. Marie, X. Qiao, X. Zhang, X. Wu, H. Fan, P. Tan, T. Amand et al., Phys. Rev. B 88, 121301 (2013).

[50] K. He, C. Poole, K. F. Mak, and J. Shan, Nano Lett. 13, 2931 (2013).

[51] M. K. L. Man, S. Deckoff-Jones, A. Winchester, G. Shi, G. Gupta, A. D. Mohite, S. Kar, E. Kioupakis, S. Talapatra, and K. M. Dani, Sci. Rep. 6, 20890 (2016).

[52] Z. Huang, C. He, X. Qi, H. Yang, W. Liu, X. Wei, X. Peng, and J. Zhong, J. Phys. D 47, 075301 (2014). 